\title{
Halite nucleation from aqueous solution
}

\author{
Aaron R. FinneY* AND MAtTeO Salvalaglio
}

Department of Chemical Engineering, University College London, Torrington Place, London WC1E 7JE, United Kingdom.

(*correspondence: a.finney@ucl.ac.uk)

Alternative pathways for the production of minerals from solutions have been proposed which, seemingly, deviate from classical notions of direct crystal nucleation and growth [1]. Metastable phases often precede the most stable crystalline mineral phase and, while such pathways are often described as being 'nonclassical', their presence does not necessarily negate classical nucleation theory (CNT) to adequately describe the thermodynamics and kinetics of stepwise phase transformations. Indeed, two-step nucleation provides a conceptual framework to explain such observations [2]. This is, however, distinct from the idea that mineral phases emerge through a process of particle attachment [1].

In silico experiments [3,4] suggest that, within the limit of solution stability, single-step CNT adequately describes halite formation from constituent ions in metastable aqueous solutions. Here we apply atomistic simulations with enhanced sampling techniques to study the thermodynamics and kinetics of ion association in the same system. Our results indicate that the crystal nucleation free energy landscape is different from one predicted using a straightforward interpretation of CNT. For instance, clusters adopt a crystalline structure consistent with bulk halite only beyond a certain size threshold that is reproducible in simulations. We explain our findings within the context of a recently proposed classical approach to two-step nucleation [5]. In addition, we extend our study to the effect of interfaces on the halite nucleation mechanism. We find that graphite, a largely chemically inert surface, affects both the local concentration and structure of ions at the solid/solution interface, which influences the pathways to mineral growth.

Our results on halite-a simple but geologically and technically important system-suggest that crystallisation in minerals may occur following more complex pathways than previously thought.

[1] J. J. De Yoreo et al. (2015), Science, 349, 6247.

[2] P. G. Vekilov (2010), Nanoscale, 2, 2346-2357.

[3] N. E. R. Zimmerman et al. (2015), J. Am. Chem. Soc., 137, 13352-13361; N. E. R. Zimmerman et al. (2018), J. Chem. Phys., 148, 222838.

[4] H. Jiang et al. (2018), J. Chem. Phys., 148, 044505; H. Jiang et al. (2019), J. Chem. Phys., 150, 124502.

[5] D. Kashchiev (2020), J. Cryst. Growth, 530, 125300. 